\title{
Effects and Mechanism of Huannao Yicong Decoction Extract on the Ethology of Transgenic APP/PS1 Mice
}

\author{
Meixia Liu, ${ }^{1}$ Yun Wei, ${ }^{1}$ Yang Yang, ${ }^{1}$ Longtao Liu, ${ }^{1}$ Lin Liang, ${ }^{2}$ Jiangang Liu, ${ }^{1}$ and Hao Li ${ }^{1}$ \\ ${ }^{1}$ Xiyuan Hospital, China Academy of Chinese Medical Sciences, Beijing 100091, China \\ ${ }^{2}$ Beijing Hospital of Traditional Chinese Medicine, Beijing 100010, China \\ Correspondence should be addressed to Jiangang Liu; liujiangang2002@sina.com and Hao Li; xyhplihao1965@126.com
}

Received 25 June 2017; Accepted 11 September 2017; Published 20 December 2017

Academic Editor: Gunhyuk Park

Copyright ( 2017 Meixia Liu et al. This is an open access article distributed under the Creative Commons Attribution License, which permits unrestricted use, distribution, and reproduction in any medium, provided the original work is properly cited.

\begin{abstract}
To investigate the mechanism of Huannao Yicong Decoction (HYD) extract on improving of learning memory of transgenic amyloid precursor protein (APP)/presenilin 1 (PS1) mice, we randomly divided 60 transgenic APP/PS1 mice of 3 months old into 4 groups: the model group, the Donepezil group, the HYD-L group, and the HYD-H group, with 15 C57BL/6J mice of the same genetic background as the control group. These mice were gavaged for 6 months in a row. The results showed that the latency was significantly shortened and the number of passing through the original platform was increased. HYD extract can increase the amount of neurons and improve the morphological structure of Nissl body obviously. The $\gamma$-secretase activity and the expression of phosphorylated APP, $\mathrm{A} \beta 1-40$, and $\mathrm{A} \beta 1-42$ in hippocampal CA1 were significantly decreased. The expressions of protein and mRNA of PEN-2 and CREB in hippocampal were significantly downregulated. These results demonstrated that HYD extract can improve the memory ability of transgenic APP/PS1 mice, which was related to the protection of neurons and structure of Nissl body, reducing cleavage of APP and production of $A \beta$ and inhibiting the activity of $\gamma$-secretase by decreasing CREB activity because of downregulated expression of PEN-2.
\end{abstract}

\section{Introduction}

The aging of population has become an irreversible trend in 21st Century. With memory and cognitive impairment as the main manifestation of Alzheimer's disease (AD) and its incidence rising, it is a serious threat to the health and life quality of elderly people. Composing of Radix polygonum multiflorum preparata, Panax ginseng, Acorus gramineus, Coptis chinensis, and Ligusticum wallichii, Huannao Yicong Decoction (HYD) is a clinical protocol, which is used to treat the elderly patients with cognitive impairment and Alzheimer's disease in Xiyuan Hospital of China Academy of Chinese Medical Sciences and has obtained the national invention patent [1] and has been applied in clinic for more than 10 years. The previous clinical studies had confirmed its effect on improving memory and cognition [2]. Basic research showed that HYD can improve the behavior of $\mathrm{AD}$ model rats, pathological morphology, and cholinergic system, inhibit the apoptosis of nerve cells, regulate the protein expression related to APP and $\mathrm{A} \beta$, and inhibit inflammatory factors and have the effect of antioxidation [3]. This study is mainly to investigate the mechanism of HYD extract on improving the learning memory of transgenic amyloid precursor protein (APP)/presenilin 1 (PS1) mice as animal model simulating $\mathrm{AD}$ through the detection of ethology, pathological morphology, and related indicators of $\gamma$-secretase.

\section{Materials and Methods}

2.1. Animals. 60 transgenic APP/PS1 mice and 15 C57BL/6J mice of a congenic genetic background, 3 months old, clean grade (SPF grade), male and female combination, weight (25-35) g, were obtained from Chinese Academy of Medical Sciences (license number: SCXK (Beijing) 2009-0007). The experiment was carried out after 7 days of adaptive feeding in the room where the temperature was controlled at $23^{\circ} \mathrm{C} \sim 25^{\circ} \mathrm{C}$ and the humidity was 50\% 70\%.

2.2. Drugs and Reagents. Donepezil hydrochloride (Aricept, $5 \mathrm{mg} /$ tablet, batch number: 100806A) was produced by 
Eisai Pharmaceutical Co. Ltd. (China). HYD extract (3.2 g crude drug/g) was prepared in the drug preparation room, Beijing University of Chinese Medicine. Chinese herbal pieces, including Radix polygonum multiflorum preparata, Panax ginseng, Acorus gramineus, Coptis chinensis, and Ligusticum wallichii, were obtained from Hebei Shennong Pharmaceutical Co. Ltd. (Beijing). Rabbit anti-phosphorylation APP/ABPP (Thr668) (batch number: 990182w), Rabbit antiA $\beta 1-40$ (CT) (batch number: 20120810), Rabbit anti-A $\beta 1-$ 42 (batch number: YE0907), Immunohistochemical staining kit (batch number: 999098), and DAB staining kit (batch number: 980579) were purchased from Beijing Bioss Technology Co. Ltd. Rabbit anti-anterior pharynx defective protein-1 (APH-1) (model: AB9214, Millipore), Rabbit anti-hypoxia inducing factor-1a (HIF-1a) (model: ab51608, Abcam), Rabbit anti-presenilin enhancer-2 (PEN-2) (model: SC-32946, Santa), Rabbit anti-cyclic adenosine monophosphate response element binding protein (CREB) (model: 06-863, Millipore), Mouse anti- $\beta$-actin antibody (model: TDY041, TiandeYue), Goat anti-rabbit IgG $(\mathrm{H}+\mathrm{L})$ (model: s004, FanxingBoao), and Luciferase assay kits (product code: E1910) were purchased from Promega company. Ultrapure RNA extraction kit (product code: CW0581), HiFi-MMLV cDNA first chain synthesis kit (product code: CW0744), Real Super Mixture (with Rox) (product code: CW0767), and DNase 1 (product code: CW2090) were purchased from Beijing Kangwei century biological technology Co. Ltd.

2.3. HYD Extract Preparation. The first step is to extract the volatile oil from Acorus gramineus, after which the drug liquid and residue together with Radix polygonum multiflorum preparata were extracted with water and precipitated with alcohol, and then the alcohol deposit liquid was obtained. Panax ginseng, Coptis chinensis, and Ligusticum wallichii were extracted together with alcohol to get the alcohol extract. The alcohol deposit liquid and alcohol extract were rotary evaporated together and then were mixed with the volatile oil, form which the final HYD extract was prepared. The HYD extract was detected by high performance liquid chromatography (HPLC) according the best chromatographic conditions which were the wavelength of $203 \mathrm{~nm}$ and $280 \mathrm{~nm}$, mobile phase of acetonitrile $-0.05 \%$ phosphoric acid water, Apollo C18 $(150 \mathrm{~mm} \times 4.6 \mathrm{~mm})$, column temperature $30^{\circ} \mathrm{C}$, and the flow rate of $1.0 \mathrm{ml} / \mathrm{min}$. The main effective components of HYD extract include ferulic acid, stilbene glycoside, berberine hydrochloride, ginseng saponin Rg1, Rbl, and $\mathrm{Re}$, and emodin whose concentrations were 2.4183, 0.8765, $1.0424,0.3993,0.5816,0.6932$, and $0.8045 \mathrm{mg} / \mathrm{g}$, respectively, reaching the requirements of Chinese Pharmacopoeia (2010 version). The quality was controlled with the combination of qualitative and quantitative methods, and the corresponding fingerprint was established (Figure 1).

2.4. Animal Grouping and Treatments. 60 transgenic APP/ PS1 mice were randomly (applying SPSS software) divided into 4 groups with 15 in each: model group (given the equal volume of distilled water), Donepezil group (given the Donepezil $0.65 \mathrm{mg} / \mathrm{kg} / \mathrm{d}$ which is clinical equivalent dose), the HYD-L group $(1.7 \mathrm{~g} / \mathrm{kg} / \mathrm{d}$ which is clinical equivalent

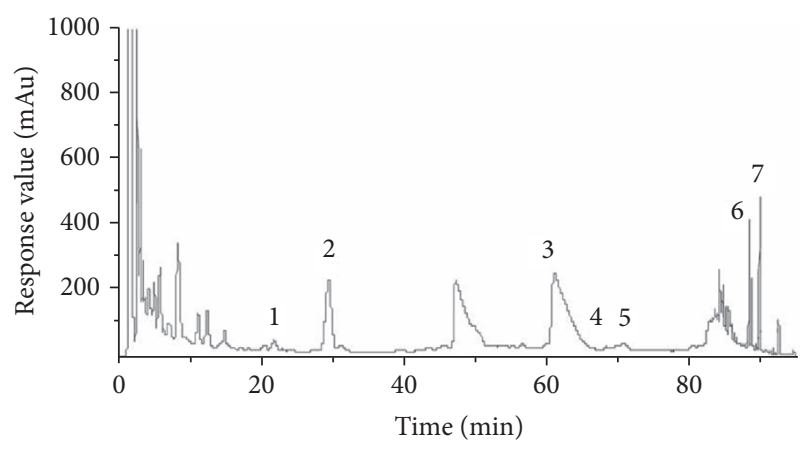

FIGURE 1: Fingerprint map of HYD extract detected with HPLC. (1) Ferulic acid; (2) styrene glycosides; (3) berberine hydrochloride; (4) ginseng saponin Rg1; (5) ginseng saponin Rbl; (6) ginseng saponin Re; (7) emodin.

dose), and the HYD-H group $(3.4 \mathrm{~g} / \mathrm{kg} / \mathrm{d}$ which is twice the clinical equivalent dose), with 15 of C57BL/6J mice as control group (given the equal volume of distilled water). The mice of the five groups were gavaged for 6 months in a row, with the liquids of the equal volume and corresponding concentration diluted by distilled water. There were 6 mice dead for the gavage reason during the experiment, 2 in the Donepezil group, 1 in HYD-L group, and 3 in HYD-H group. The experiment was in accordance with the animal ethics requirements.

2.5. Morris Water Maze Test. The Morris water maze test was carried out after 6-month gavage, to assess the learning memory ability of the transgenic APP/PS1 mice. The Morris water maze apparatus $(120 \mathrm{~cm}$ in diameter and $50 \mathrm{~cm}$ in depth) was produced by Institute of Pharmacology, Chinese Academy of Chinese Medicine. The water in the pool was $30 \mathrm{~cm}$ deep and approximately $1 \mathrm{~cm}$ above the platform surface, which was located in the fourth quadrant, with the temperature of $25 \pm 1^{\circ} \mathrm{C}$. Some milk powder was added in the water to make it opaque white, making the mice (black) unable to distinguish the platform in the tank. The whole experiment was divided into two parts: place navigation test and spatial probe test. For the place navigation test, at the marked entry point from the second quadrant $1 / 2$ radian of the pool wall, the mice were put into the water against the pool wall one by one. Record the time (name as escape latency) the mice spend finding the platform (until the limbs on the platform) and the swimming distance by an autotracking system. Animals were allowed to swim for $3 \mathrm{~min}$ to search for the platform at each trial and to take a rest on platform for $10 \mathrm{~s}$ if they found it. For those animals who failed the mission, the escape latency was recorded as $180 \mathrm{~s}$. All the mice were trained once a day and for 4 consecutive days. On the fifth day, the escape latency as an evaluation index of the learning memory ability of mice was recorded in the morning. In the afternoon the spatial probe test was performed to assess the spatial memory ability with removal of the platform. Choose an entry point, put the mice into the water with mice facing the pool wall, and record the times of crossing the platform-site in $3 \mathrm{~min}$.

All mice were sacrificed by decapitation after the Morris water maze test. The brain was removed immediately after the 
transcardial perfusion with saline. The brains were sagittally cut into brain specimens of left or right sides allocated randomly on the ice. Six specimens in each group were fixed in $4 \%$ neutral paraformaldehyde solution and were sliced after paraffin embedded, which were used to observe after Nissl Staining and immunohistochemistry. The hippocampi of other specimens were isolated and stored in refrigerator at $-80^{\circ} \mathrm{C}$, in order to detect the related molecules by dualluciferase reporter, Western blotting, and real-time fluorescent quantitative-polymerase chain reaction (RT-PCR).

2.6. Nissl Staining. The paraffin-embedded brain slices were deparaffinized and washed routinely, washed 1-2 min with distilled water, dipped for $30 \mathrm{~min}$ in $1 \%$ thionin water solution at $37^{\circ} \mathrm{C}$ incubator, then washed $1-2 \mathrm{~min}$ with distilled water, differentiated to see clear Nissl body with 95\% alcohol, dehydrated for 8-10 min with anhydrous alcohol, permeabilized for 8-10 min with xylene, and sealed with neutral gum. Neurons and Nissl bodies in hippocampus CA1 area were observed by $\mathrm{BH}-2$ biological microscope (produced by Olympus of Japan).

2.7. Immunohistochemistry. All paraffin-embedded brain slices were deparaffinized routinely and washed with distilled water, dipped in PBS for $5 \mathrm{~min}$, incubated for $20 \mathrm{~min}$ in $3 \% \mathrm{H}_{2} \mathrm{O}_{2}$ deionized water to eliminate the endogenous peroxidase activity, and incubated for $20 \mathrm{~min}$ at room temperature after working solution of normal goat serum was dropped for sealing; then the liquid was poured out with no washing. Then the slices were incubated overnight at $4^{\circ} \mathrm{C}$ with primary antibodies, which were diluted by appropriate proportion. On the second day, the slices were incubated at room temperature for $20 \mathrm{~min}$ with the secondary antibodies, before and after which the slices were washed 3 times with PBS (each for $3 \mathrm{~min}$ ). After incubation at room temperature for $20 \mathrm{~min}$ with the working solution of horseradish peroxidase labeled streptavidin, the slices were washed 3 times with PBS, each for 3 min, stained with DAB, and then washed fully with tap water, redyed, dehydrated, transparentized, and sealed finally. The hippocampus CA1 area of mice was observed with $\mathrm{BH}-2$ biological microscope. The positive result was brown yellow cytoplasm or prominence. Two slices from each mouse were observed and taken pictures of, applying the Image Pro Plus 6.0 image analysis software (produced by American Media Cybernetics corporation) to calculate the average optical density (OD) value of positive expression in each high power $(\times 600)$ view.

2.8. Dual-Luciferase Reporter. Five hippocampus tissue specimens in each group were chosen and grinded with $200 \mathrm{ul}$ lysates of luciferase assay kits, centrifuged for $5 \mathrm{~min}$ with the speed of $15000 \mathrm{~g}$. Then $20 \mathrm{ul}$ of the supernatant was sucked out, which was blended with 100 ul Luciferase Assay Buffer II, The luciferase value was read using multifunctional microplate reader (produced by Molecular Devices corporation). Then $100 \mathrm{ul}$ of stop\&Glo Buffer was added and mixed well; then the Renilla value was recorded. Luciferase/Renilla value indicated $\gamma$-secretase activity with Renilla value as internal reference.
2.9. Western Blotting. Six samples in each group were used to extract the tissue proteins and protein quantifying by BCA kit. According to the loading quantity of sample of sodium dodecyl sulfate polyacrylamide gel electrophoresis (SDS-PAGE), the protein concentrations were adjusted with RIPA and $5 x$ buffer solution of restored sample and the protein solution were boiled for $5 \mathrm{~min}$. According to the molecular weights of target proteins, separation gels of $8 \%$ and $12 \%$ were prepared, respectively; the concentration of spacer gel was made at 5\%. For electrophoresis, spacer gel constant voltage was set at $90 \mathrm{v}$ for about $20 \mathrm{~min}$. Separation gel constant voltage was set at $160 \mathrm{v}$, and the stop time of the electrophoresis was determined by predyed marker protein. Then the proteins were transferred onto the membranes, sealed, and incubated with primary antibodies and the membranes were washed. The proteins were incubated with the internal reference, which was $\beta$ eta-actin mouse monoclonal antibody diluted with 3\% BSA-TBST (rate 1:5000), for $2 \mathrm{~h}$ at room temperature. After incubating with the secondary antibody, the membranes were washed again. After the ECL reaction, the film was exposed, developed, and fixed.

2.10. RT-PCR. Six samples in each group were selected and the total RNA were extracted with ultrapure RNA extraction kit. Electrophoresis was performed on $5 \mathrm{ul}$ of the RNA liquid with $1 \%$ agarose gel with DYY-GC electrophoresis apparatus (produced by Beijing Liuyi Instrument Factory). Primers were synthesized by Beijing Huateng Shengwei Biological Technology Co. Ltd., with $\beta$-actin as internal reference. The upstream of APH-la mRNA primer was $5^{\prime}$-ATGCACCTTCGTCGCGTTCG-3' and the downstream was $5^{\prime}$-AGAAGGACAGAGACAGCAGCACCAA-3' (amplified length was $222 \mathrm{bp}$ ). The upstream of HIF- $1 \alpha$ mRNA primer was $5^{\prime}$-GATGAATCAAAAACAGAGACGAAGG-3' and the downstream was $5^{\prime}$-CTGATGCCTTAGCAGTGGTCGT-3' (amplified length was $109 \mathrm{bp}$ ). The upstream of PEN-2 mRNA primer was $5^{\prime}$-ACACAGAGCAGAGCCAAATCAAAGG-3 $3^{\prime}$ and the downstream was $5^{\prime}$-CAGGGGAATGGTGAAGGAGAGGTAG-3' (amplified length was $155 \mathrm{bp}$ ). The upstream of CREB mRNA primer was $5^{\prime}$-ACCATTGCCCCTGGAGTTGTTAT- $3^{\prime}$ and the downstream was $5^{\prime}$-CTCTTGCTGCCTCCCTGTTCTTC-3' (amplified length was $115 \mathrm{bp}$ ). The upstream of internal reference $\beta$ actin mRNA primer was $5^{\prime}$-GCCTTCCTTCTTGGGTAT- $3^{\prime}$ and the downstream was $5^{\prime}$-GGCATAGAGGTCTTTACGG$3^{\prime}$ (amplified length was $97 \mathrm{bp}$ ). Reverse transcription was conducted using the first strand synthesis kit of HiFi-MMLV cDNA and the amplification was conducted with RealSuper Mixture (with Rox) with the procedure of $95^{\circ} \mathrm{C}$ for $10 \mathrm{~min}$, 40 cycles with melting for $15 \mathrm{~s}$ at $95^{\circ} \mathrm{C}$ and annealing and extension for $1 \mathrm{~min}$ at $60^{\circ} \mathrm{C}$. The melting curve was analyzed at $60-95^{\circ} \mathrm{C}$, after which the standard curve of the target gene and internal reference gene was drawn. At last the data was relatively quantified and analyzed using the method of $2^{-\Delta \Delta \mathrm{CT}}$.

2.11. Statistical Analyses. The data were expressed as the mean \pm SEM. All statistical analyses were done by using the statistical package of SPSS 17.0. One-way ANOVA and 


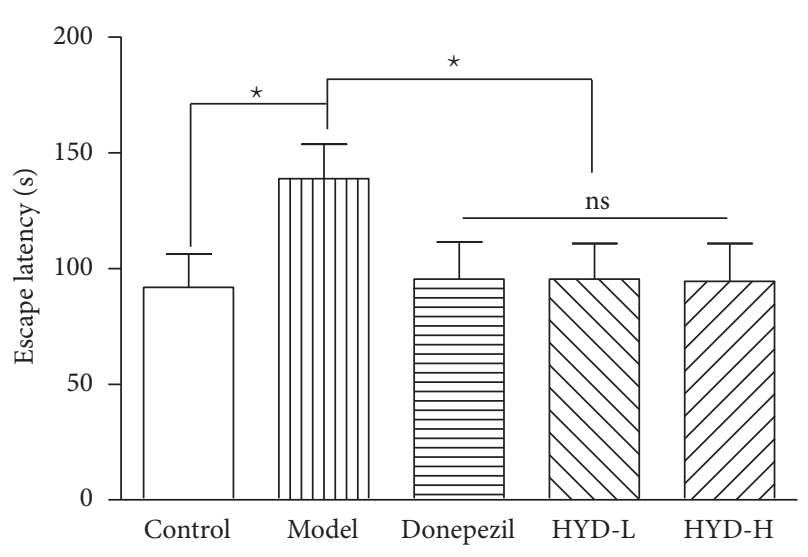

(a)

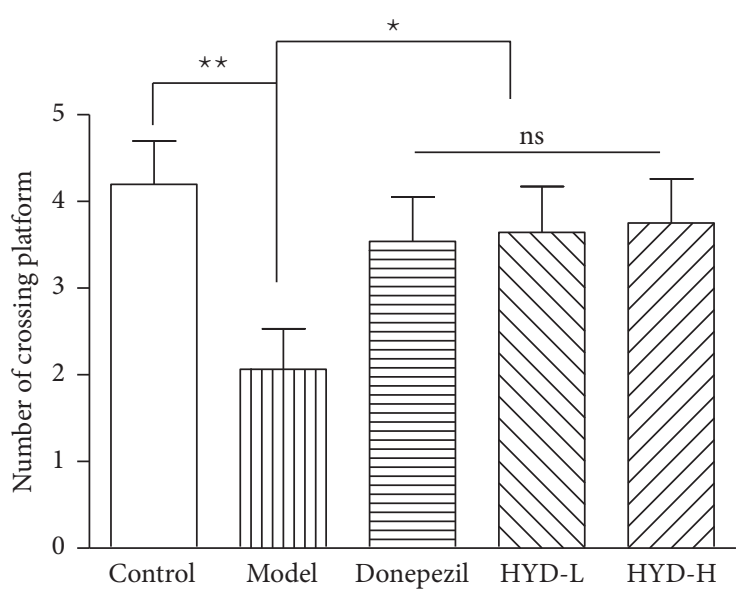

(b)

FIGURE 2: HYD extract improved the ability of learning and memory of transgenic APP/PS1 mice. (a) Escape latency, (b) number of crossing platform. ${ }^{\star} P<0.05,{ }^{\star \star} P<0.01$.

q-test were used to compare among groups after all the data were tested with the normality test and homogeneity test of variance. $P$ values of less than 0.05 were considered significant and those less than 0.01 were considered obviously significant.

\section{Results}

3.1. Effects of HYD Extract on the Learning Memory Ability of Transgenic APP/PS1 Mice. As shown in Figure 2, compared with the control group, the escape latency of model group mice was significantly prolonged $(P<0.05)$; the number of crossing platform was decreased significantly $(P<0.01)$; after intervention, compared with the model group, the latencies of Donepezil group, HYD-L group, and HYD-H group were shortened $(P<0.05)$; the number of crossing platform was increased $(P<0.05)$ (Figure 2$)$.

\subsection{Effects of HYD Extract on Neurons and Nissl Body} of Hippocampal CA1 Area of Transgenic APP/PS1 Mice. As shown in Figure 3, with Nissl Staining, the neurons of control group mice in hippocampus CA1 area were in a larger quantity, arranged in neat rows, rich in Nissl bodies (dark blue), with pale blue nucleus and light blue background; the neurons of model group mice in the hippocampal CA1 area decreased in quantity, arranged disorderedly, with obscure or disappeared boundary of Nissl bodies, karyopyknosis, or karyolysis which were shaped in elliptic or triangle and dyed dark blue; the neurons of Donepezil group, HYD-L group, and HYD-H group mice in the hippocampal CA1 area increased significantly in quantity, arranged neatly, with more Nissl bodies, little karyopyknosis, and karyolysis (Figure 3).

3.3. Effects of HYD Extract on the Expression of APP and $A \beta$ in Hippocampal CA1 Area of Transgenic APP/PS1 Mice. Compared with the control group, the expressions of phosphorylated APP and A $\beta$ of model control group mice in hippocampal CA1 area were significantly upregulated
$(P<0.01)$; after drug administration, compared with the model group, the expression of phosphorylated APP and A $\beta 1-42$ of Donepezil group, HYD-L group, and HYD-H group and the expression of phosphorylated A $\beta 1-40$ of HYDL group, and HYD-H group were significantly downregulated $(P<0.01)$; and the expressions of phosphorylated APP and A $\beta 1-40$ of the HYD-H group were significantly lower than the Donepezil group and the HYD-L group $(P<0.01)$ (Figures 4(a), 4(b), and 4(c)).

3.4. Effects of HYD Extract on the $\gamma$-Secretase Activity of Transgenic APP/PS1 Mice. Compared with the control group, the $\gamma$-secretase activity of model group significantly increased $(P<0.01)$; after drug administration, compared with the model group, the $\gamma$-secretase activity of HYD-L group and HYD-H group decreased $(P<0.05)$; compared with the Donepezil group, the $\gamma$-secretase activity of HYD-H group decreased $(P<0.05)$ (Figure 5).

3.5. Effects of HYD Extract on the Protein Expression of APH1a, HIF-1 $\alpha, P E N-2$, and CREB of Transgenic APP/PS1 Mice. Compared with the control group, the protein expressions of APH-1a, HIF-1 $\alpha$, PEN-2, and CREB were increased significantly $(P<0.05, P<0.01)$; after drug administration, compared with the model group, the protein expressions of PEN2 and CREB of HYD-L group and HYD-H group significantly decreased $(P<0.05, P<0.01)$; compared with the Donepezil group, the protein expressions of PEN-2 of HYD-L group and HYD-H group decreased $(P<0.05)$ (Figures 6(a), 6(b), 6(c), and $6(\mathrm{~d}))$.

3.6. Effects of HYD Extract on the mRNA Expression of APH1a, HIF-1 $\alpha$, PEN-2, and CREB of Transgenic APP/PS1 Mice. Compared with the control group, the mRNA expression of APH-1a, HIF-1 $\alpha$, PEN-2, and CREB of model group increased significantly $(P<0.05, P<0.01)$; after drug administration, compared with the model group, the mRNA expression of 

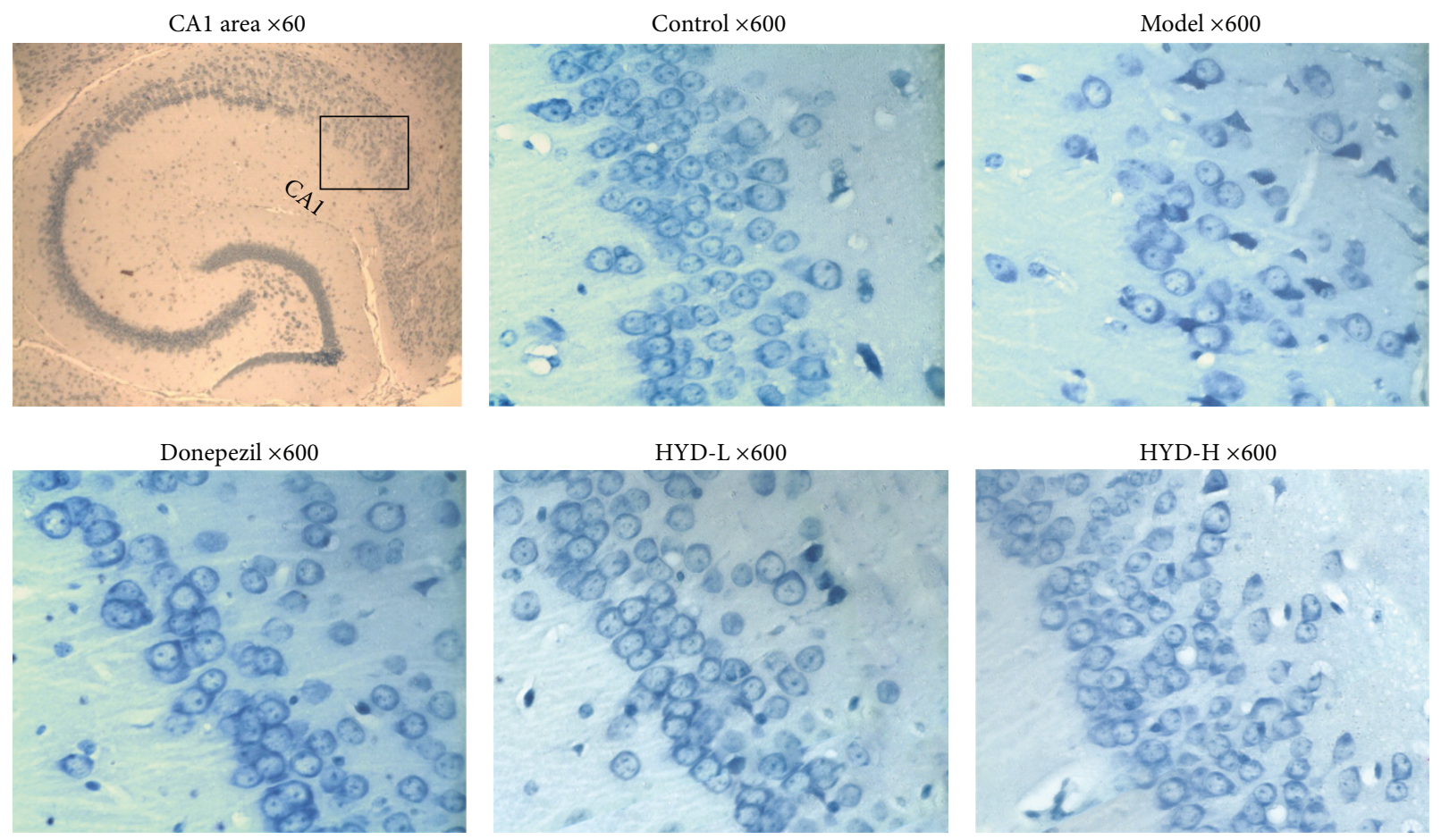

FIGURE 3: HYD extract protected the neuron and Nissl body of transgenic APP/PS1 mice (Nissl Staining, $\times 60$ and $\times 600$ ).

PEN-2 of HYD-L group and the mRNA expression of PEN2 and CREB of HYD-H group significantly decreased $(P<$ $0.05, P<0.01)$; compared with the Donepezil group, the mRNA expression of PEN-2 of HYD-L group and HYD-H group significantly decreased $(P<0.05, P<0.01)$ (Figures 7(a), 7(b), 7(c), and 7(d)).

\section{Discussion}

Alzheimer's disease is a neurodegenerative disease characterized by progressive memory decline and cognitive impairment. The main pathological features of Alzheimer's disease include senile plaques, nerve fiber entanglement, and neuronal loss. The production and deposition of $\mathrm{A} \beta$ (especially for $A \beta 1-42$ ) play an important role in the pathological process. The $\mathrm{A} \beta$ deposition in extracellular space directly results in the senile plaques and then further induces tau protein hyperphosphorylation in the cells, forming the neurofibrillary tangles, both of which can cause the corresponding neurotoxic effects, eventually making the structure and function of neurons injured and lost $[4,5] . A \beta$ is produced through hydrolyzing of amyloid precursor protein (APP) by $\beta$ and $\gamma$ secretase. The $\gamma$-secretase is the final key of the formation of $A \beta$, whose activity is closely related to the occurrence of Alzheimer's disease. So the intervention in above pathological process is very important for the prevention and treatment of Alzheimer disease [6].

APP mutation can cause the structural change of APP binding sites with $\beta$-secretase, which increased $\beta$-secretase activity, so as to increase the amount of $\mathrm{A} \beta$, and intensifies the formation of senile plaques. PS1 mutation can change $\gamma$ secretase activity, resulting in the change of the metabolic process of APP, selectively causing A $\beta 1-42$ production and increase. So the transgenic APP/PS1 mice can well simulate human Alzheimer's disease of graduated progress [7]. According to the reports, the 2.5-month-old mice began to show $\mathrm{A} \beta$ deposition, around 3-month-old mice began to show cognitive and behavioral changes of early stage, 7-month-old mice showed cognitive impairment, and 9month-old mice showed dysfunction of spatial memory [810]. The pathological mechanism of the model is associated with activation of $\gamma$-secretase. It is an ideal model for the study of drugs in the regulation of $\gamma$-secretase pathway and is also a successful transgenic AD model domestically recognized [11].

$\gamma$-secretase is kind of membrane integrated protease complexes with large molecular weight, which is composed of four subunits: presenilin (PS, catalytic activity center of $\gamma$ secretase), nicastrin (NCT, the assembly bracket and binding site of substrate of $\gamma$-secretase), anterior pharynx defective1 (APH-1, stent partner of NCT), and presenilin enhancer2 (Pen-2, the assembly molecular wedge of $\gamma$-secretase complex). The expression changes of the four essential proteins play an important role in $\gamma$-secretase activity, the production of $\mathrm{A} \beta$, and the onset of $\mathrm{AD}$ [12-16]. The research group studied all the four proteins. This thesis mainly summarizes the research results of APH-1 and PEN-2 and their related factors and the research results of NCT and PS were published in Chinese Journal of Pharmacology and Toxicology [17].

APH-1 is one of the essential constituent proteins of $\gamma$ secretase in mammalian cells, with two highly homologous proteins: APH-la and APH-lb. APH-la is the main isomer of $\gamma$-secretase in the process of embryonic development, and lacking APH-la can reduce the production of A $\beta$. HIF-1 is a transcription regulatory factor of APH-1a, which is composed 


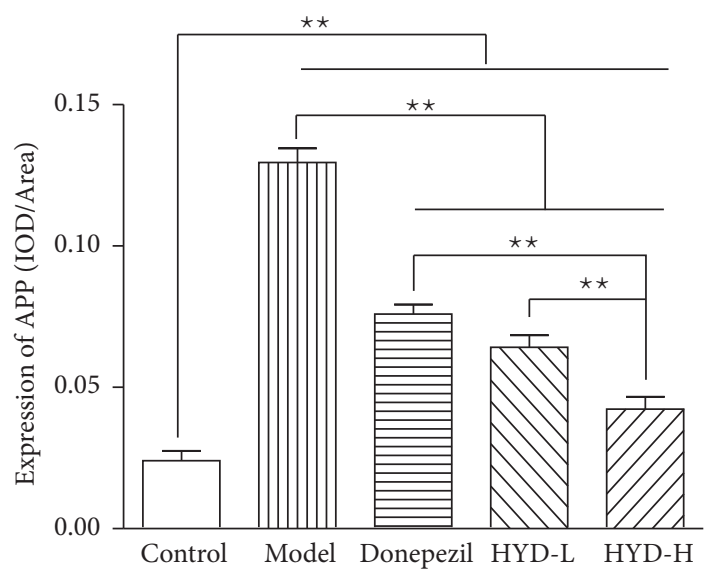

CA1 area $\times 60$

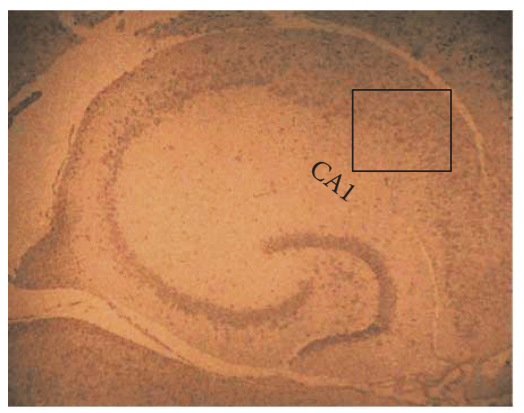

Donepezil $\times 600$

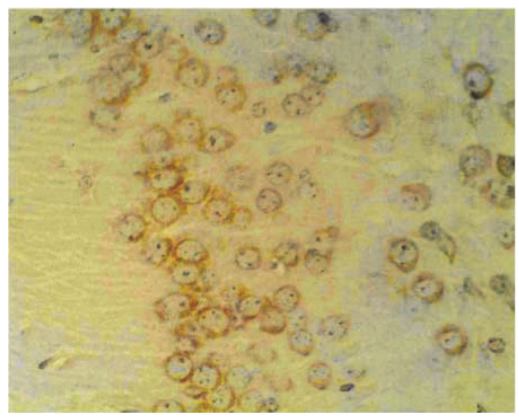

Control $\times 600$

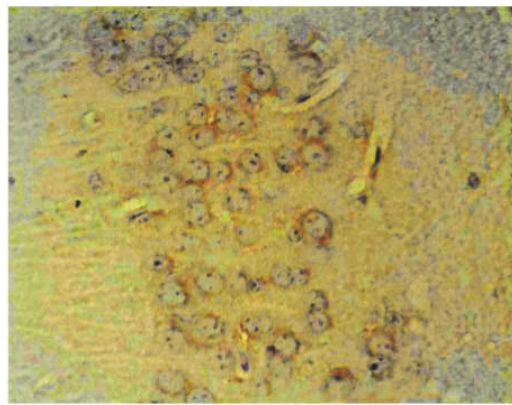

HYD-L ×600

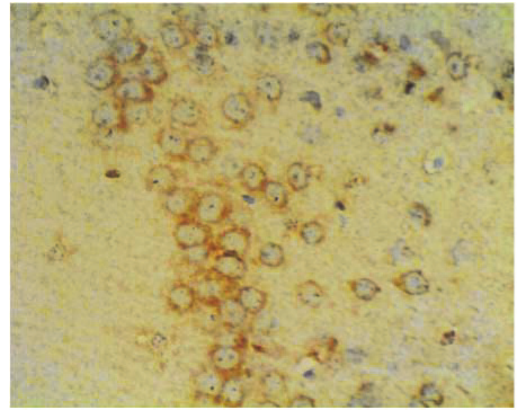

(a)

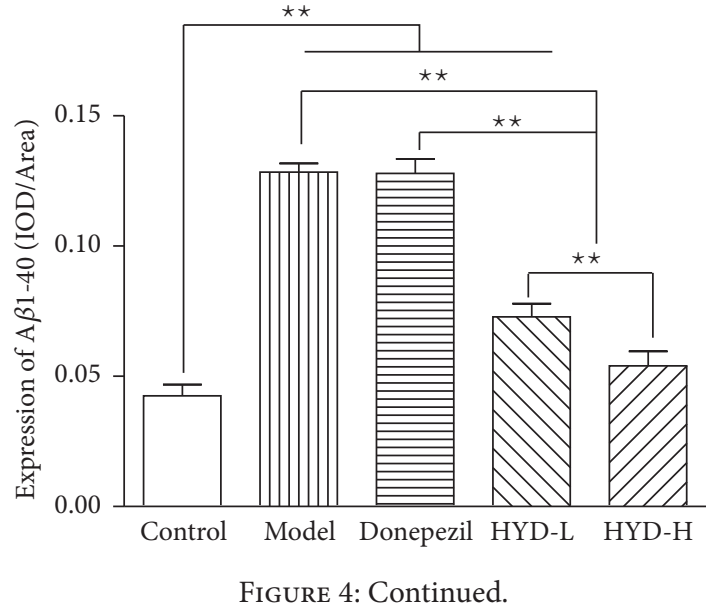

Model ×600

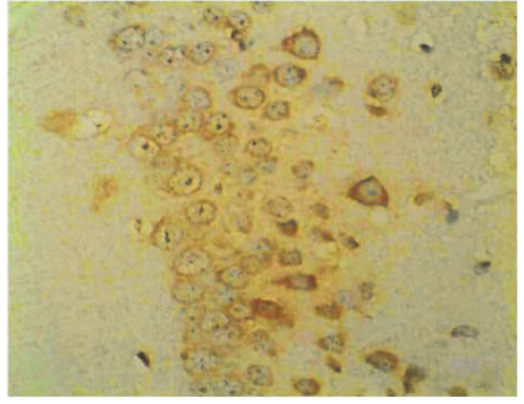

HYD-H ×600

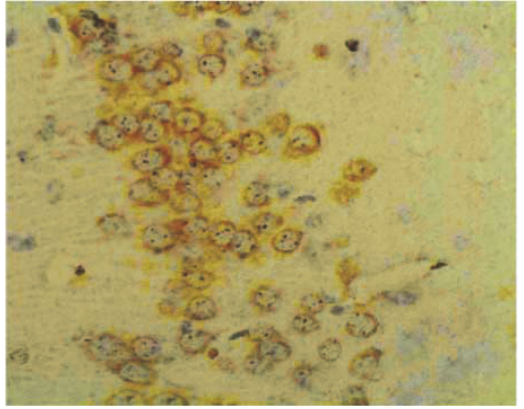


CA1 area $\times 60$

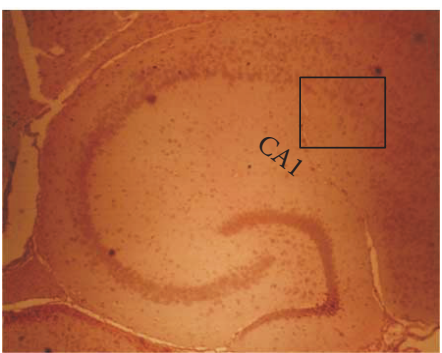

Donepezil $\times 600$

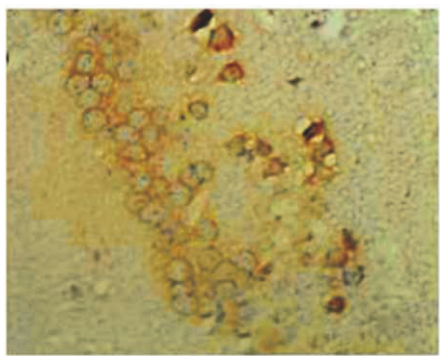

Control $\times 600$

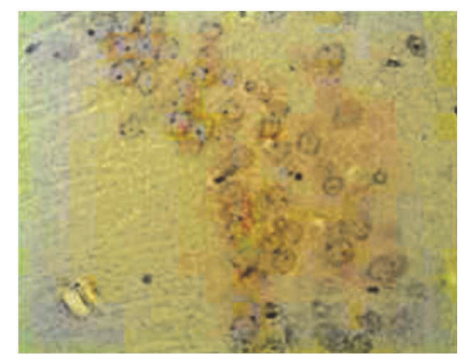

HYD-L $\times 600$

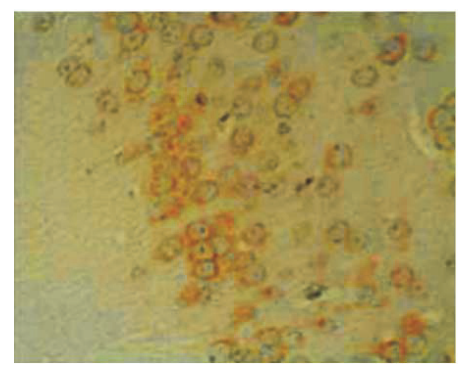

(b)
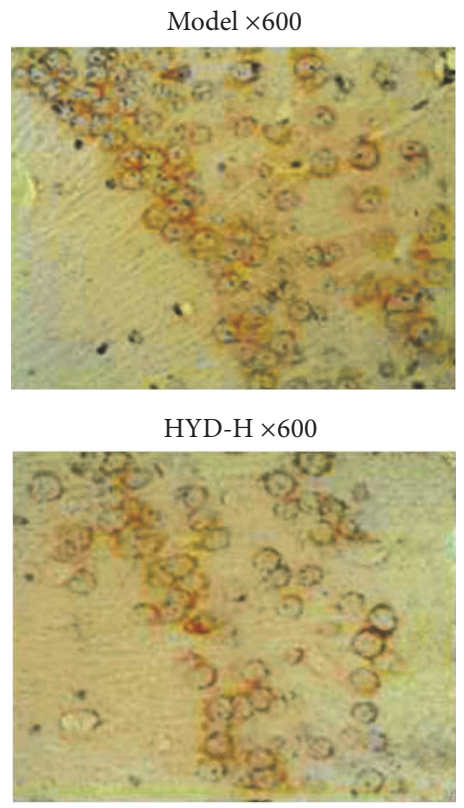

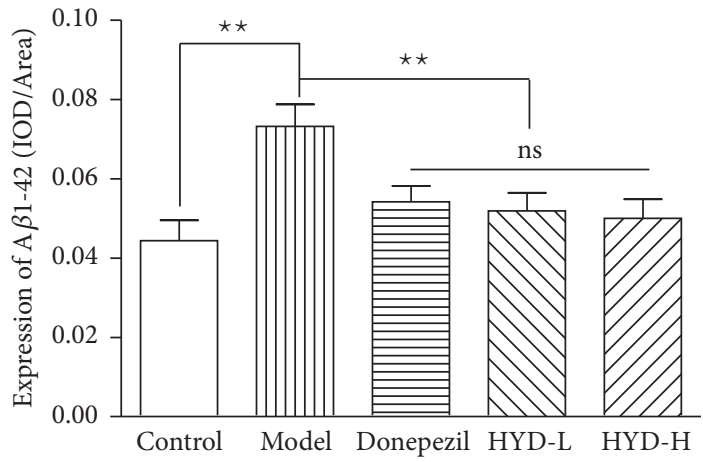

CA1 area $\times 60$

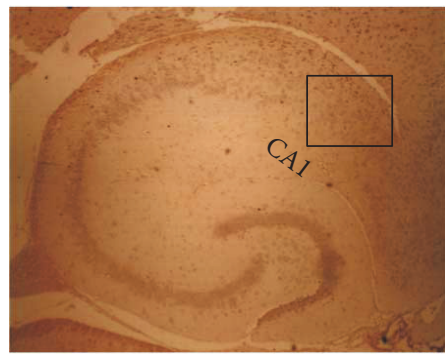

Donepezil $\times 600$

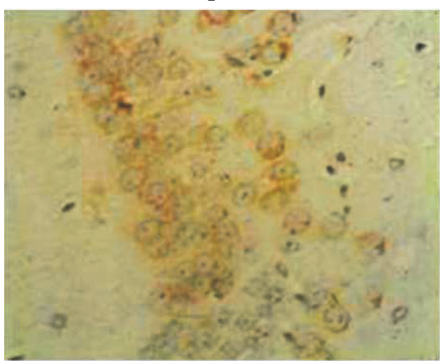

Control $\times 600$

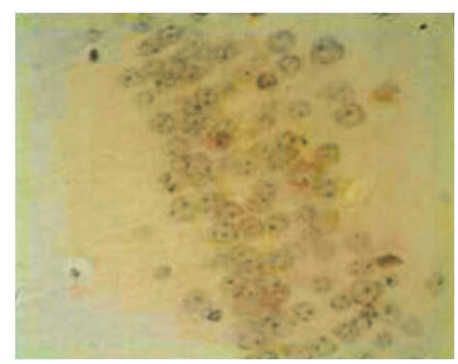

HYD-L $\times 600$

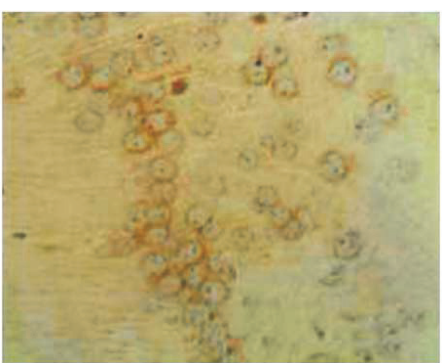

(c)

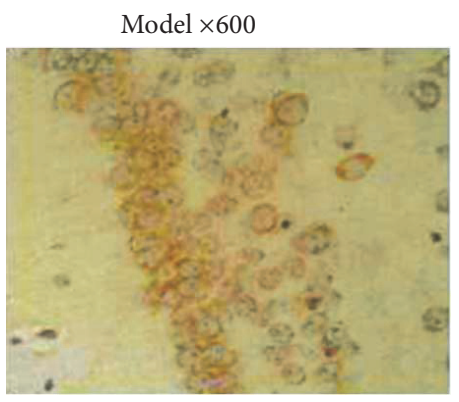

HYD-H $\times 600$

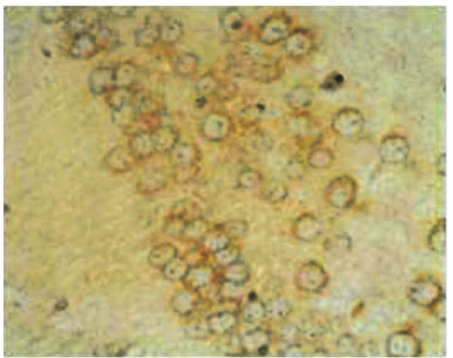

FIgURE 4: HYD extract regulated protein expression of APP, A $\beta 1-40$, and A $\beta 1-42$ in the hippocampus CA1 area of transgenic APP/PS1 mice (immunohistochemistry, $\times 60$ and $\times 600$ ). (a), (b), and (c) are the protein expressions of APP, A $\beta 1-40$, and $\mathrm{A} \beta 1-42 .{ }^{\star \star} P<0.01$. 


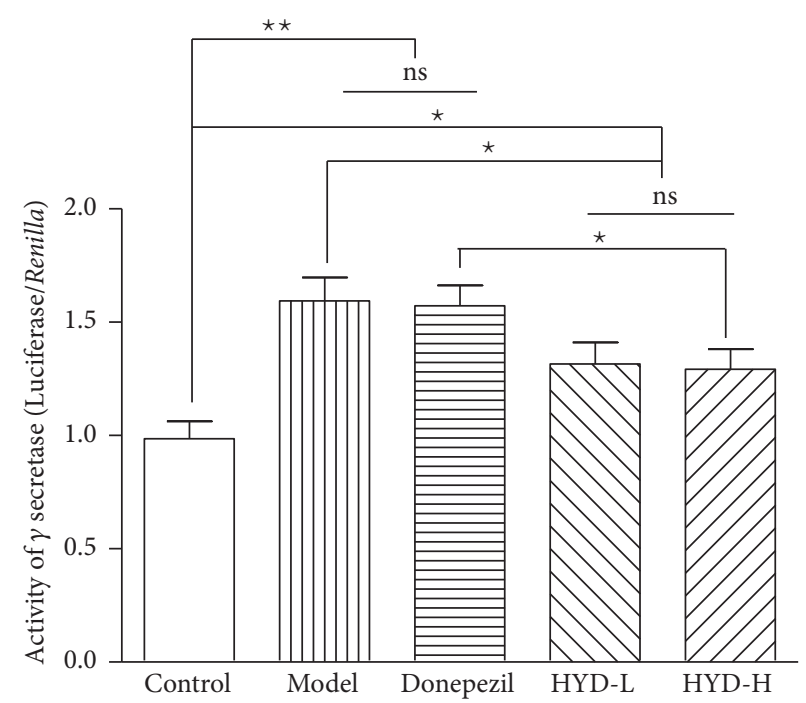

FIGURE 5: HYD extract regulated activity of $\gamma$-secretase of transgenic APP/PS1 mice. ${ }^{\star} P<0.05,{ }^{\star \star} P<0.01$.

of subunits HIF- $1 \alpha$ and HIF- $1 \beta$. The physiological activity of HIF-1 mainly depends on the activity and expression of HIF$1 \alpha[18,19]$. Studies had found that APH-la promoter contains the binding sites of HIF-1, and when HIF-1 is activated, the levels of mRNA and protein of APH-1a, $\gamma$-secretase activity and $\mathrm{A} \beta$ production are significantly increased [20].

PEN-2 is widely expressed in specific nerve cells of most brain areas of transgenic APP/PS1 mice, including the olfactory bulb, basal ganglia, striatum, cortex, hippocampus, amygdala, thalamus, hypothalamus, cerebellum, brainstem, and spinal cord. It is not only the main component of $\gamma$-secretase, but also the modulator of $\gamma$-secretase activity, playing a key role in the production of $A \beta 1-42$ [21-23]. CREB, a transcription regulatory factor of $\mathrm{PEN}-2$, which can activate some specific genes to produce proteins, strengthens the connection between cells and is closely related to the formation and maintaining of long-term memory [24]. The study found that the promoter of PEN-2 gene contains CREB binding site and can bind to each other specifically, consequently decreasing $\gamma$-secretase activity and $\mathrm{A} \beta$ formation through inhibiting CREB activity and reducing the expression of PEN-2 [25].

The results of this study show that the latency of 9month-old transgenic APP/PS1 mice in the Morris water maze test was significantly prolonged, and the platform-site crossing times were significantly decreased, which showed that the spatial learning and memory ability were significantly decreased. On the pathological morphology, neurons and Nissl body in hippocampal CA1 area were significantly decreased in quantity and significantly impaired in structure. Meanwhile, $\gamma$-secretase activity and the expression of APP, $\mathrm{A} \beta 1-40$, and $\mathrm{A} \beta 1-42$ in hippocampus $\mathrm{CA1}$ area were significantly increased. Furthermore, the APH-la, HIF- $1 \alpha$, PEN2, CREB protein, and their mRNA expression of 9-monthold transgenic APP/PS1 mice were significantly increased. It suggested that HIF- $1 \alpha$ binding sites of APH-la promoter and CREB binding sites of PEN-2 promoter in the hippocampal tissue of transgenic APP/PS1 mice were activated, which resulted in a significant increase in APH-1a and PEN-2 expression and $\gamma$-secretase activity and caused APP dissociation and production of a large number of $\mathrm{A} \beta$. Neurons damage and loss were caused by further accumulation of $\mathrm{A} \beta$, which affected the spatial learning and memory. It was one of the main reasons for the mice to become AD model.

In Traditional Chinese medicine theory, $\mathrm{AD}$ is thought as amnesia or dementia which was caused by deficiency of the kidney essence in origin and blood stasis and turbidity in superficiality. So our team proposed that treatment regimens of $\mathrm{AD}$ were reinforcing deficiency and supplementing body essence mainly and promoting blood circulation to remove blood stasis and relieving toxicity and transforming turbid secondly. HYD was formulated through above regimen that was composed of Radix polygonum multiflorum preparata, Panax ginseng, Acorus gramineus, Coptis chinensis, and Ligusticum wallichii. Early toxicity experiments had confirmed that HYD extract was safe and had no obvious side effects.

After drug administration of six months, HYD extract can significantly shorten the latency and increase the crossing times of transgenic APP/PS1 mice in Morris water maze test; can obviously protect neurons and Nissl body, improve their quantity and structure, and promote the protein synthesis in hippocampus CA1 area; can significantly increase $\gamma$-secretase activity and the expression of APP, $A \beta 1-40$, and A $\beta 1-42$; can obviously decrease PEN-2, CREB protein, and their mRNA expression and its effect is better than or equivalent to Donepezil. The experiment showed that HYD extract can down-regulate PEN-2 expression and $\gamma$-secretase activity through inhibiting the CREB binding site activation of PEN-2 gene promoter, intervene in APP metabolic pathways, reduce the production of $\mathrm{A} \beta 1-40$ and $\mathrm{A} \beta 1-42$, restrain the formation of senile plaques, reduce the loss of neurons, improve memory, and then inhibit AD occurring and developing. So it had a positive meaning for early applying HYD extract to prevent and treat AD. However, HYD extract could not obviously change APH-la, HIF- $1 \alpha$ protein, and their mRNA expression, which indicated that APH-la and HIF- $1 \alpha$ are not HYD targets in treating $\mathrm{AD}$. 
Control Model Donepezil HYD-L HYD-H
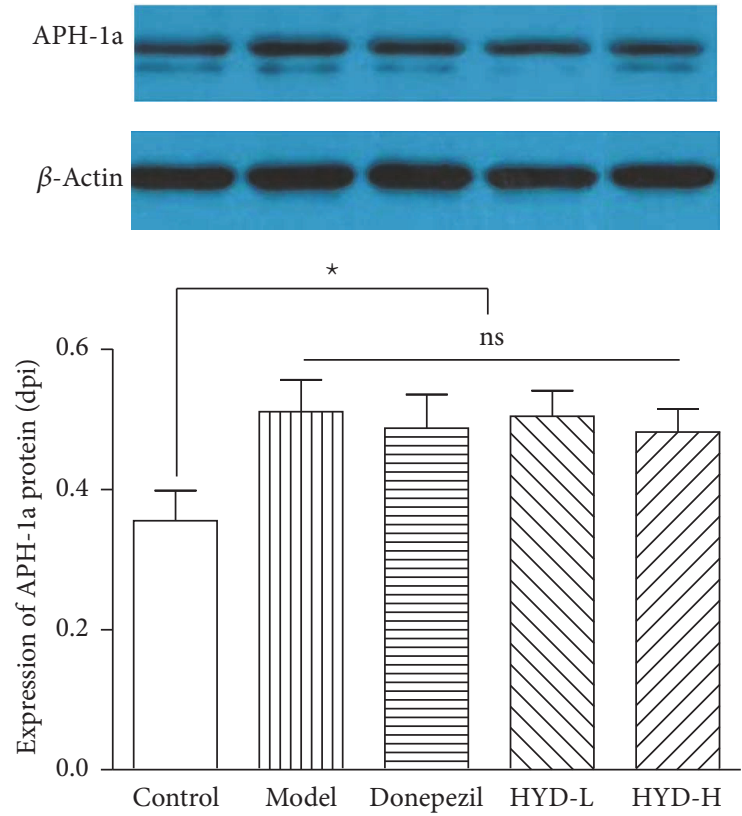

(a)
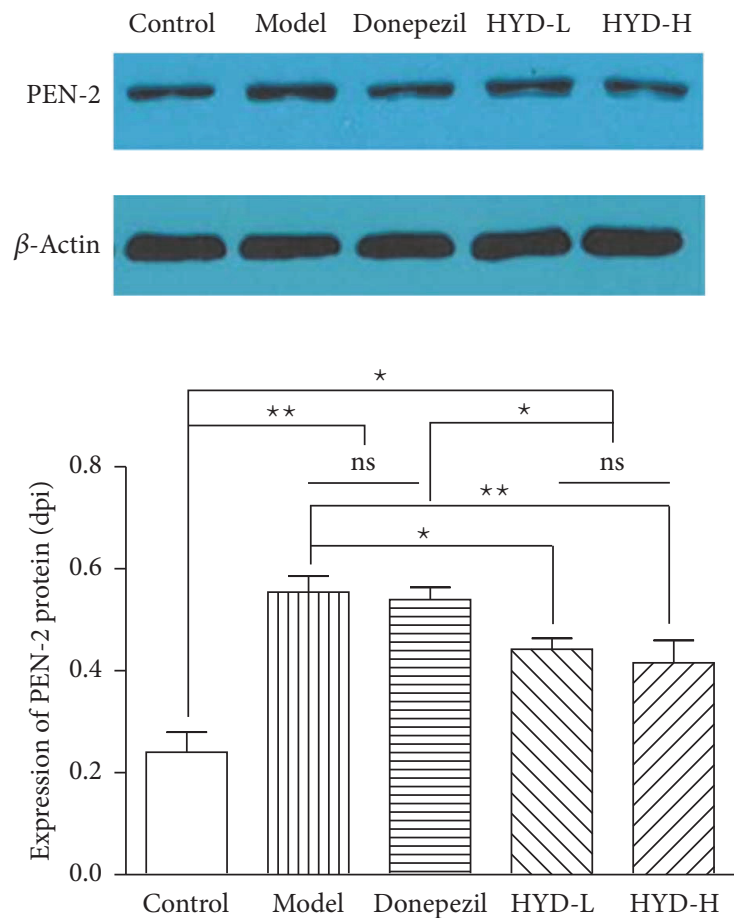

(c)
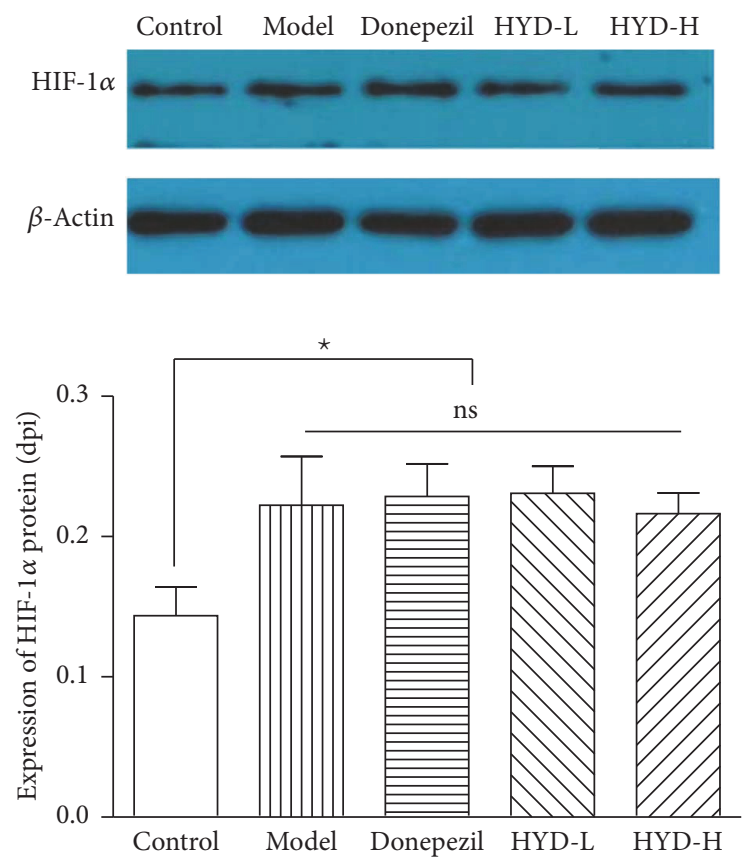

(b)
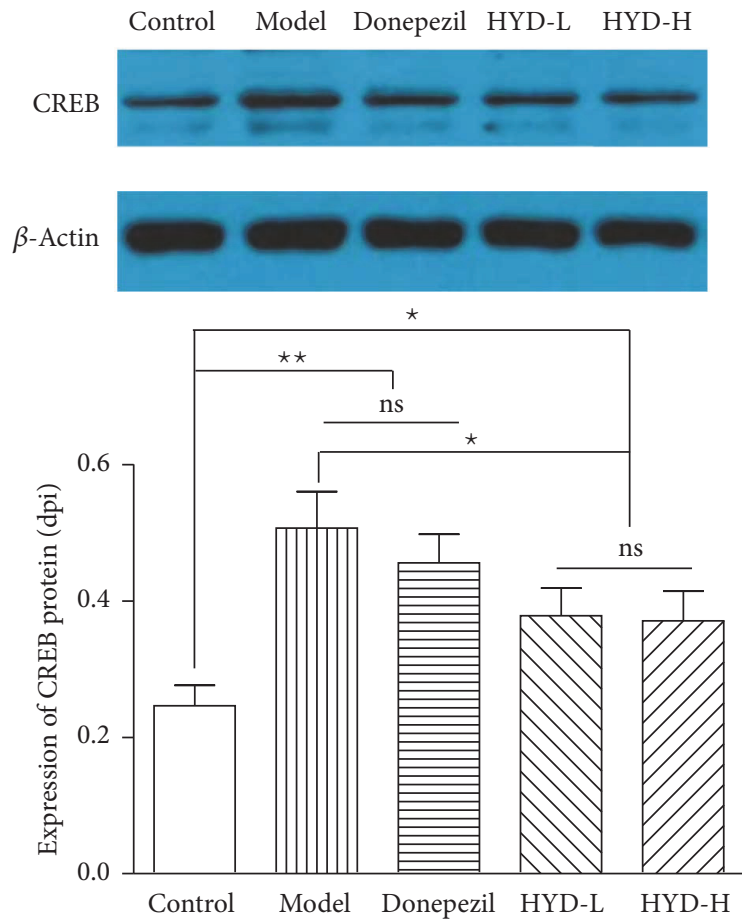

(d)

FIGURE 6: HYD extract regulated protein expression of APH-1a, HIF-1 $\alpha$, PEN-2, and CREB of transgenic APP/PS1 mice (Western blotting, APH-1a 1:1000, HIF-1 $\alpha 1: 1000$, PEN-2 1:200, CREB 1:2000, $\beta$-actin $1: 5000)$. (a), (b), (c), and (d) are the protein expressions of APH-la, HIF- $1 \alpha$, PEN-2, and CREB. ${ }^{\star} P<0.05,{ }^{\star \star} P<0.01$. 


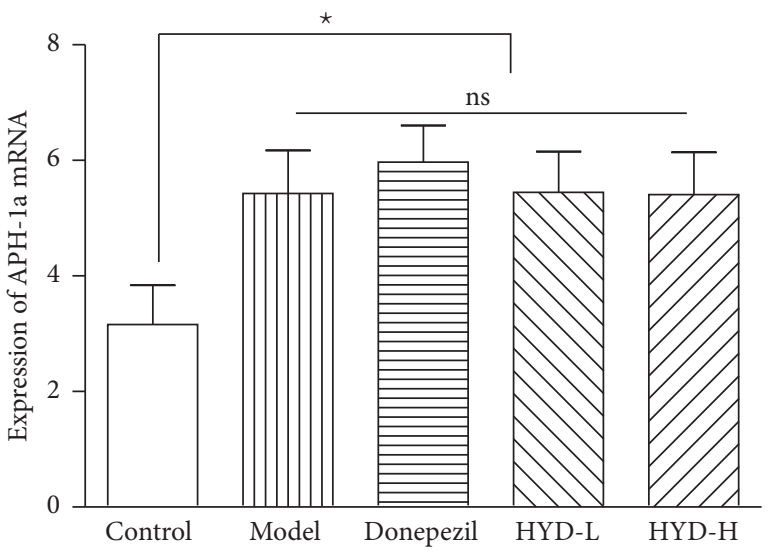

(a)

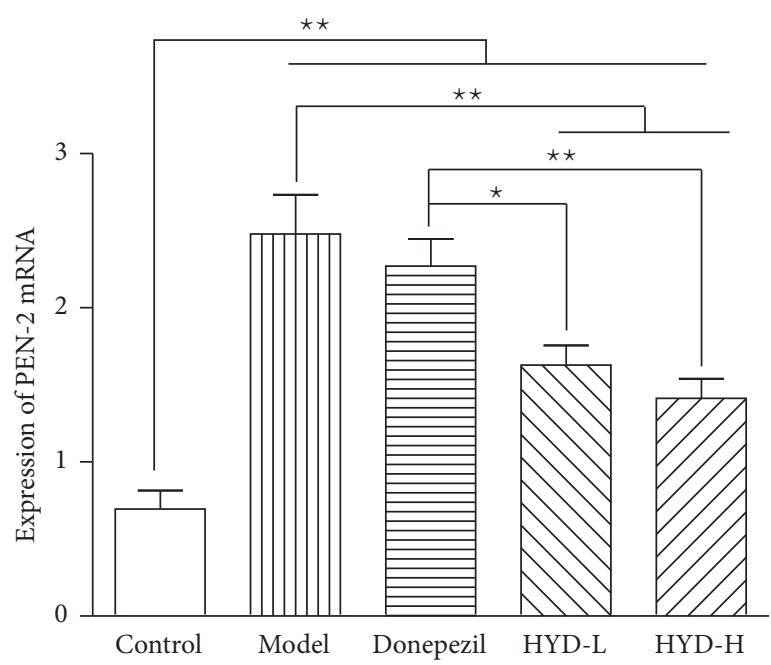

(c)

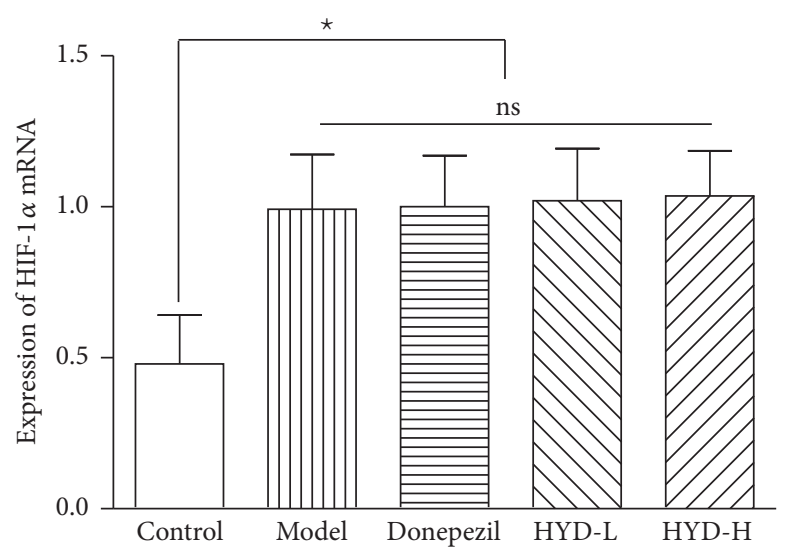

(b)

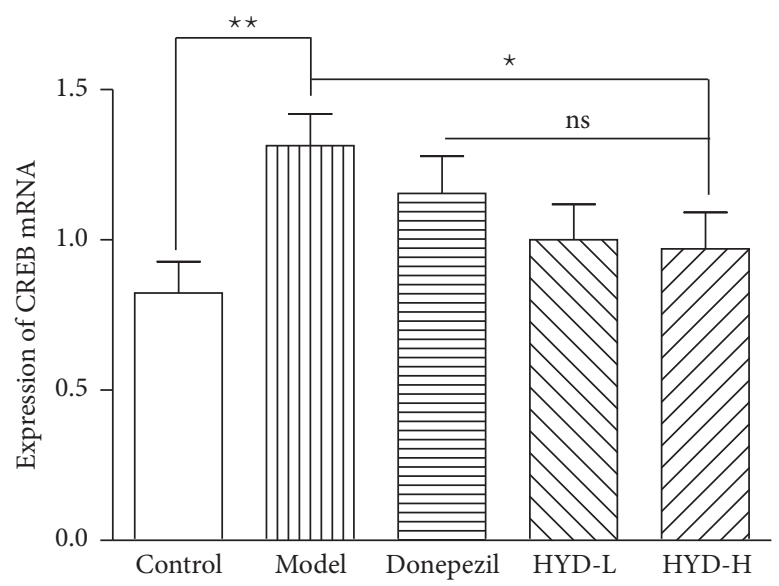

(d)

FIGURE 7: HYD extract regulated mRNA expression of APH-la, HIF-1 $\alpha$, PEN-2, and CREB of transgenic APP/PS1 mice. (a), (b), (c), and (d) are the mRNA expressions of APH-1a, HIF-1 $\alpha$, PEN-2, and CREB. ${ }^{\star} P<0.05,{ }^{\star \star} P<0.01$.

\section{Conclusion}

HYD extract can obviously improve the spatial learning and memory ability of transgenic APP/PS1 mice. The mechanism was related to its protection of neurons and the structure of Nissl body, the reduction of APP cleavage and A $\beta$ (especially $\mathrm{A} \beta 1-42$ ), and the inhibition of $\gamma$-secretase activity which results from the inhibition of CREB activity and further downregulated expression of PEN-2.

\section{Disclosure}

The funders had no role in study design, data collection and analysis, decision to publish, or preparation of the paper.

\section{Conflicts of Interest}

All the authors of this paper declare that they do not have a direct financial relation with the commercial identities mentioned in the paper.

\section{Authors' Contributions}

Hao Li and Jiangang Liu designed the experiments; Meixia Liu, Yun Wei, Yang Yang, Longtao Liu, Lin Liang, Jiangang Liu, and Hao Li performed the experiments, analyzed the data, and wrote the manuscript; Meixia Liu and Yun Wei equally contributed to the paper.

\section{Acknowledgments}

The authors acknowledge financial support from the National Natural Science Foundation of China (no. 81173383, no. 81573819, and no. 81503628), the National Important Specialized Science and Technology Item of Science and Technology Department, China (no. 2009ZX09103-391), and a Science and Technology Project of Beijing, China (no. Z141100002214003).

\section{References}

[1] H. Li, J. G. Liu, L. T. Liu, M. X. Liu, Y. Wei, and J. Guan, "kind of Traditional Chinese medicine for preventing and treating 
Alzheimer's disease and its preparation method," Chinese Patent, 2011.

[2] H. Li, M.-J. Yao, W.-M. Zhao, J. Guan, L.-L. Cai, and L. Cui, "A randomized, controlled, double-blind trial of Huannao Yicong capsule in senile patients with mild cognitive impairment," Journal of Chinese Integrative Medicine, vol. 6, no. 1, pp. 25-31, 2008.

[3] M. X. Liu, J. G. Liu, H. Li, and W. Q. Zhou, "Research ideas and methods of Chinese herbal medicine for preventing and treating senile dementia based on a variety of animal models," World Science and Technology/Modernization of Traditional Chinese Medicine and Materia Medica, vol. 14, no. 2, pp. 13671375, 2012.

[4] J. M. Nussbaum, M. E. Seward, and G. S. Bloom, "Alzheimer disease: A tale of two prions," Prion, vol. 7, no. 1, pp. 14-19, 2013.

[5] X. Li, F. Guo, Q. Zhang et al., "Electroacupuncture decreases cognitive impairment and promotes neurogenesis in the APP/PS1 transgenic mice," BMC Complementary and Alternative Medicine, vol. 14, article 37, 2014.

[6] Z. Zhou, C. H. Chan, Q. Ma, X. Xu, Z. Xiao, and E. Tan, "The roles of amyloid precursor protein (APP) in neurogenesis," Cell Adhesion \& Migration, vol. 5, no. 4, pp. 280-292, 2014.

[7] T. M. Wengenack, S. Whelan, G. L. Curran, K. E. Duff, and J. F. Poduslo, "Quantitative histological analysis of amyloid deposition in Alzheimer's double transgenic mouse brain," Neuroscience, vol. 101, no. 4, pp. 939-944, 2000.

[8] Y. Y. Zong, X. Y. Wang, H. L. Wang et al., "Continuous analysis of senile plaque and behaviourin APP/PS double-transgenic gene mouse model of alzheimer disease," Chin J CompMed, vol. 18, no. 9, pp. 8-14, 2008.

[9] A. J. Bruce-Keller, S. Gupta, A. G. Knight et al., "Cognitive impairment in humanized APP $\times$ PS1 mice is linked to A $\beta 1-42$ and NOX activation," Neurobiology of Disease, vol. 44, no. 3, pp. 317-326, 2011.

[10] S. J. Webster, A. D. Bachstetter, and L. J. van Eldik, "Comprehensive behavioral characterization of an APP/PS-1 double knockin mouse model of Alzheimer's disease," Alzheimer's Research \& Therapy, vol. 5, no. 3, article 28, 2013.

[11] I. Hussain, D. C. Harrison, J. Hawkins et al., "TASTPM mice expressing amyloid precursor protein and presenilin-1 mutant transgenes are sensitive to $\gamma$-secretase modulation and amyloid$\beta 42$ lowering by GSM-10h," Neurodegenerative Diseases, vol. 8, no. 1-2, pp. 15-24, 2010.

[12] W. T. Kimberly, M. J. LaVoie, B. L. Ostaszewski, W. Ye, M. S. Wolfe, and D. J. Selkoe, " $\gamma$-secretase is a membrane protein complex comprised of presenilin, nicastrin, Aph-1, and Pen-2," Proceedings of the National Acadamy of Sciences of the United States of America, vol. 100, no. 11, pp. 6382-6387, 2003.

[13] H. Steiner, "The catalytic core of $\gamma$-secretase: Presenilin revisited," Current Alzheimer Research, vol. 5, no. 2, pp. 147-157, 2008.

[14] S. Shah, S.-F. Lee, K. Tabuchi et al., "Nicastrin functions as a $\gamma$ secretase-substrate receptor," Cell, vol. 122, no. 3, pp. 435-447, 2005.

[15] A. C. Chen, L. Y. Guo, B. L. Ostaszewski, D. J. Selkoe, and M. J. LaVoie, "Aph-1 associates directly with full-length and Cterminal fragments of $\gamma$-secretase substrates," The Journal of Biological Chemistry, vol. 285, no. 15, pp. 11378-11391, 2010.

[16] M. Niimura, N. Isoo, N. Takasugi et al., "Aph-1 contributes to the stabilization and trafficking of the $\gamma$-secretase complex through mechanisms involving intermolecular and intramolecular interactions," The Journal of Biological Chemistry, vol. 280, no. 13, pp. 12967-12975, 2005.
[17] M. X. Liu, J. G. Liu, H. Li et al., "Effects and mechanism of HuannaoYicongDecoction Extract on learning and memory ability of Transgenic APP/PS1 Mice," Chin J Pharmacol Toxicol, vol. 28, no. 1, pp. 10-17, 2014.

[18] K. Shirotani, D. Edbauer, S. Prokop, C. Haass, and H. Steiner, "Identification of distinct $\gamma$-secretase complexes with different APH-1 variants," The Journal of Biological Chemistry, vol. 279, no. 40, pp. 41340-41345, 2004.

[19] N. Sanjo, T. Katayama, H. Hasegawa et al., "Localization and trafficking of endogenous anterior pharynx-defective 1, a component of Alzheimer's disease related $\gamma$-secretase," Neuroscience Letters, vol. 483, no. 1, pp. 53-56, 2010.

[20] R. Wang, Y.-W. Zhang, X. Zhang et al., “Transcriptional regulation of APH-1A and increased gamma-secretase cleavage of APP and Notch by HIF-1 and hypoxia.," The FASEB journal : official publication of the Federation of American Societies for Experimental Biology, vol. 20, no. 8, pp. 1275-1277, 2006.

[21] O. Holmes, S. Paturi, D. J. Selkoe, and M. S. Wolfe, "Pen-2 is essential for $\gamma$-secretase complex stability and trafficking but partially dispensable for endoproteolysis," Biochemistry, vol. 53, no. 27, pp. 4393-4406, 2014.

[22] L. Bammens, L. Chávez-Gutiérrez, A. Tolia, A. Zwijsen, and B. De Strooper, "Functional and topological analysis of pen-2, the fourth subunit of the $\gamma$-secretase complex," The Journal of Biological Chemistry, vol. 286, no. 14, pp. 12271-12282, 2011.

[23] Y. Chu, X. Peng, Z. Long et al., "Distribution and expression of Pen-2 in the central nervous system of APP/PS1 double transgenic mice," Acta Biochimica et Biophysica Sinica, vol. 47, no. 4, pp. 258-266, 2015.

[24] M. R. Walton and M. Dragunow, "Is CREB a key to neuronal survival?" Trends in Neurosciences, vol. 23, no. 2, pp. 48-53, 2000.

[25] R. Wang, Y.-W. Zhang, P. Sun et al., “Transcriptional regulation of PEN-2, a key component of the $\gamma$-secretase complex, by CREB," Molecular and Cellular Biology, vol. 26, no. 4, pp. 13471354, 2006. 


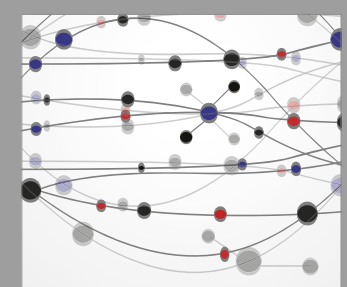

The Scientific World Journal
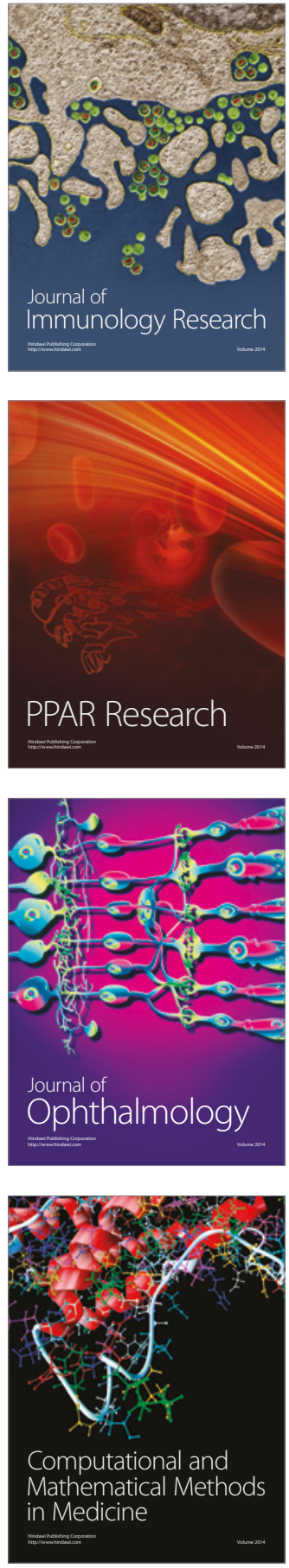

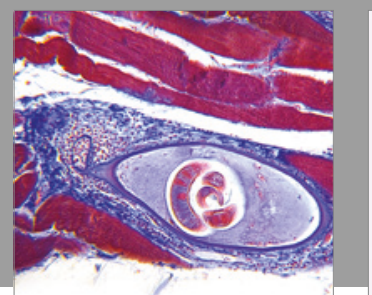

Gastroenterology Research and Practice
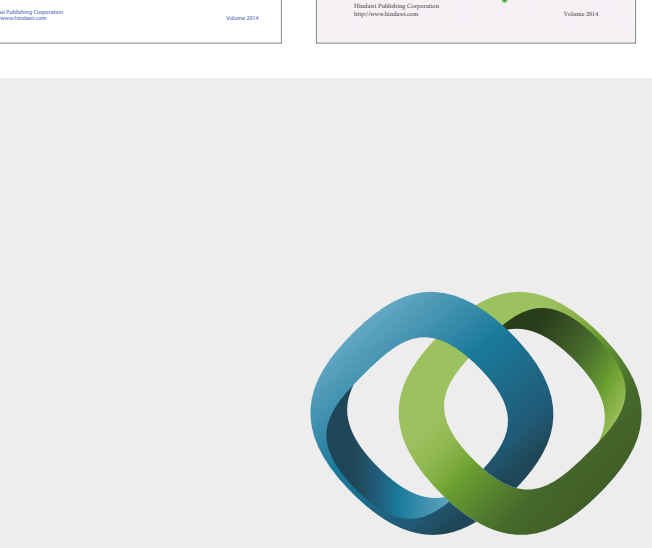

\section{Hindawi}

Submit your manuscripts at

https://www.hindawi.com
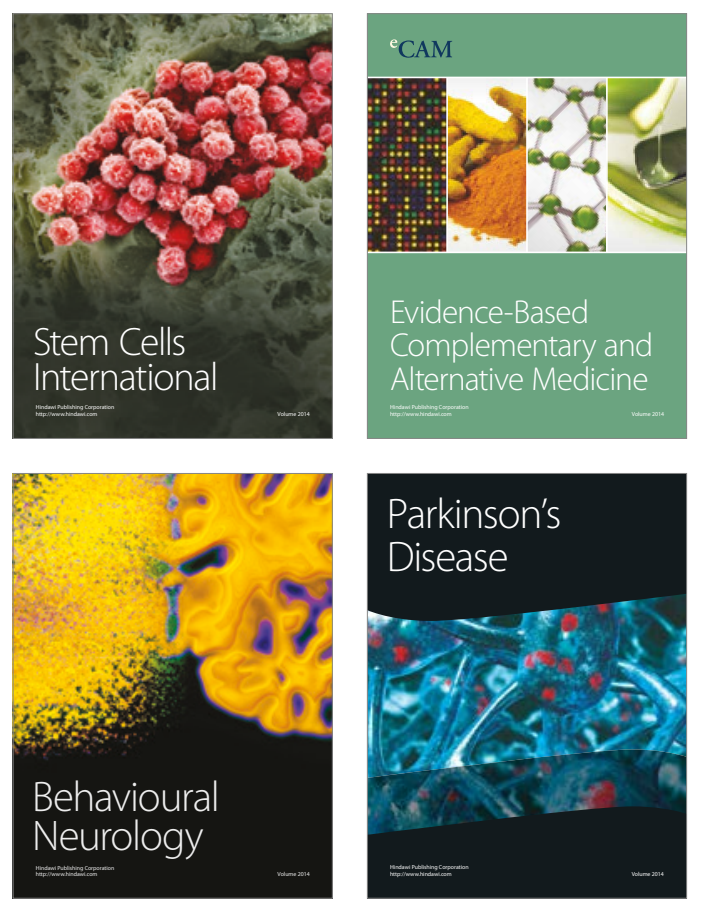
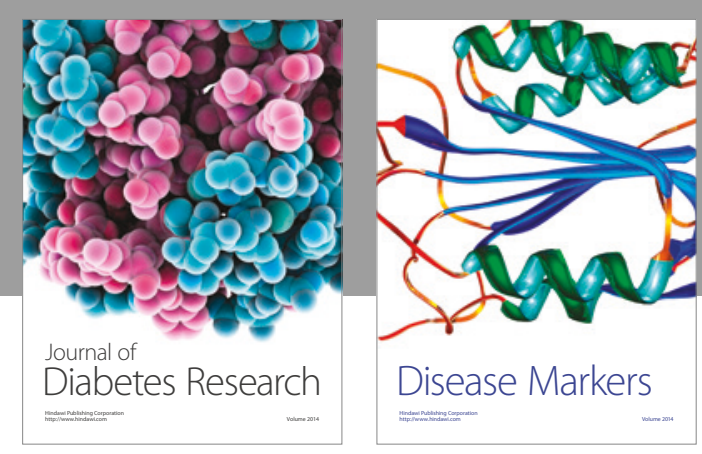

Disease Markers
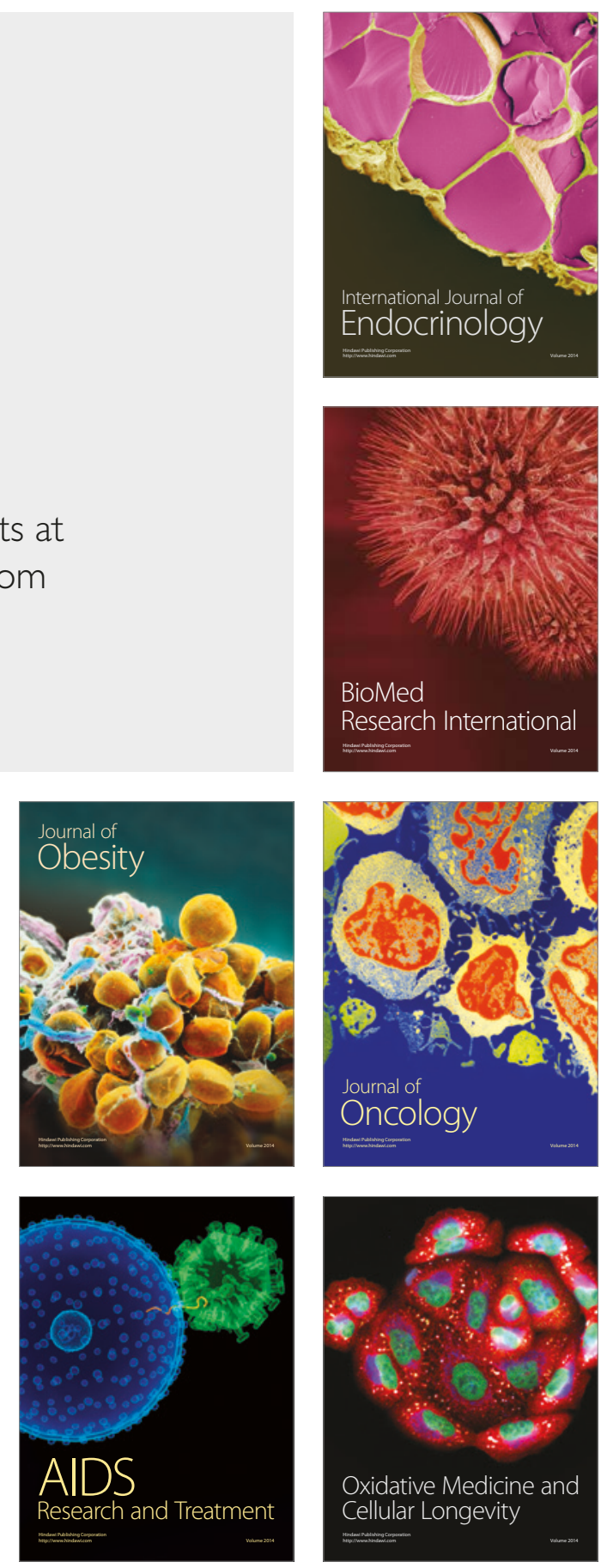